(RESEARCH ARTICLE)

\title{
GC-MS analysis of Abelmoschus manihot (L.) Medik (Malvaceae) leaves
}

\author{
Divya Selvaraj ${ }^{1}$, Arivoli Subramanian $1{ }^{*}$ and Tennyson Samuel ${ }^{2}$ \\ 1 Department of Zoology, Thiruvalluvar University, Vellore 632 115, Tamil Nadu, India. \\ ${ }^{2}$ Department of Zoology, Madras Christian College, Chennai 600 059, Tamil Nadu, India.
}

Publication history: Received on 27 January 2020; revised on 12 February 2020; accepted on 14 February 2020

Article DOI: https://doi.org/10.30574/wjarr.2020.5.2.0025

\begin{abstract}
Plants are a rich source of bioactive phytochemicals which provide health benefits for humans further than those attributed to macronutrients and micronutrients. The phytochemical compounds isolated and purified are employed in a wide range of applications. In the present study, the bioactive compounds of Abelmoschus manihot benzene, chloroform, methanol and ethanol leaf extracts via GC-MS was analyzed and its biological properties being available in pure form, being nontoxic with a wide spectrum of biological functions, may find its application in the formation of various medicinal products.
\end{abstract}

Keywords: Abelmoschus manihot; Leaf extracts; GC-MS; Phytochemical compounds

\section{Introduction}

Plants of the genus Abelmoschus belong to the family of flowering plants called Malvaceae. This genus, also known as okra is composed of numerous species of flowering plants in the mallow family and are native to tropical and subtropical areas [1]. Interest in this genus is due principally to the high protein and mineral salt content of the pods, making okra a very good vegetable. Studies have shown that the daily consumption of $100 \mathrm{~g}$ of okra provides 20, 15 and $50 \%$ calcium, iron, and Vitamin C of human dietary requirements respectively [2]. Onakpa [3] and Patil et al. [4] have documented the ethnomedicinal, phytochemical and pharmacological profile of the genus Abelmoschus. The species Abelmoschus manihot is cultivated mainly in the Far East, but also in the Indian sub-continent and northern Australia. It is less frequently found in America and tropical Africa. On the latter continent, Chevalier [5] described the variety zenkeri in Cameroon and the variety caillei in West Africa. The latter has also been observed in Zaire [6]. This species contains various chemical ingredients including flavonoids, organic acids, steroids, volatile constituents, coumarins, aliphatic hydrocarbons and nitrogenous compounds [7]. It has been used for treatment of chronic renal disease, mouth ulcers, and burns [8-10]. This plant species is also reported to possess analgesic [11], antiinflammatory [12], antiviral [13], antibacterial [14], anticoagulant [15], larvicidal [16], wound healing [12] and osteoporosis $[17,18]$ properties. In the present study, the GC-MS analysis of this plant species has been analyzed as studies reported above have shown that there is scope to use this plant as a source of medicinal agent.

\section{Material and methods}

\subsection{Plant collection and preparation of extracts}

Mature and healthy leaves of Abelmoschus manihot were located and collected from in and around of Unaivaniyambadi village, Vellore district, Tamil Nadu, India $\left(12.8730^{\circ} \mathrm{N}, 78.9714^{\circ} \mathrm{E}\right)$. Taxonomical identity of the plant was confirmed at the Department of Biotechnology, Thiruvalluvar University, Vellore, Tamil Nadu, India. Leaves were then washed under running tap water, air dried and shade dried for 10-15 days. The leaves were powdered using an electronic

\footnotetext{
${ }^{*}$ Corresponding author

E-mail address: rmsarivoli@gmail.com
} 
blender and sieved to get fine powder. The powdered leaves (500 g) were macerated with various solvents (1.5 L) each, viz., benzene, chloroform, methanol and ethanol using a Soxhlet apparatus [19] with their respective temperatures. The crude extract thus obtained was concentrated by evaporation and the yield was used for further phytochemical analysis.

\subsection{GC-MS Analysis}

GC-MS analysis was carried out at the Sophisticated Instrumentation Facility (SIF), Chemistry division, School of Advanced Science, VIT University, Vellore, Tamil Nadu, India. The Clarus 680 GC used in the analysis employed a fused silica column, packed with Elite-5MS (5\% biphenyl $95 \%$ dimethylpolysiloxane, $30 \mathrm{~m} \times 0.25 \mathrm{~mm}$ ID $\times 250 \mu \mathrm{m}$ df) and the components were separated using Helium as carrier gas at a constant flow of $1 \mathrm{~mL} / \mathrm{minute}$. The injector temperature was set at $260{ }^{\circ} \mathrm{C}$ during the chromatographic run. The extract sample $(1 \mu \mathrm{L})$ injected into the instrument with the oven temperature was as follows: $60{ }^{\circ} \mathrm{C}(2$ minutes $)$; followed by $300{ }^{\circ} \mathrm{C}$ at the rate of $10{ }^{\circ} \mathrm{C} \mathrm{min}-1$; and $300{ }^{\circ} \mathrm{C}$, where it was held for six minutes. The mass detector conditions were: transfer line temperature $240{ }^{\circ} \mathrm{C}$; ion source temperature $240{ }^{\circ} \mathrm{C}$; and ionization mode electron impact at $70 \mathrm{eV}$, a scan time 0.2 seconds and a scan interval of 0.1 seconds. The fragments were from 40 to $600 \mathrm{Da}$. The spectrums of the components were compared with the database of spectrum of known components stored in the GC-MS NIST library.

\section{Results}

The phytochemical compounds via GC-MS of the benzene leaf extract of Abelmoschus manihot indicated the presence of phytol, palmitic acid, linoleic acid, dioctyl phthalate, tocopherol, Urs-12-en-28-ol, (2E,4E)-2,4-heptadecadienoic acid and stigmast-4-en-3-one (Table 1; Figure 1). The GC-MS of chloroform extract revealed the presence of phytol, methyl isopalmitate, palmitic acid, linoleic acid, 25-hydroxycholesterol, DL- $\alpha$-tocopherol acetate, 3,5-di-tertbutylbenzaldehyde, 12-hydroxy-8,10-heptadecadienoic acid, 22,23-dibromostigmasterol acetate and cholest-4-en-3one (Table 2; Figure 2). The phytochemical compounds in the methanol leaf extract specified the presence of phytol, 2,3-dimethyl-8-oxo-non-2-enal, palmitic acid, 1,1'-bi(cyclohexyl), DL- $\alpha$-tocopherol acetate, Urs-12-en-3-ol-acetate(3ß), 12-hydroxy-8,10-heptadecadienoic acid and fludrocortisone acetate (Table 3; Figure 3). The GC-MS study of ethanol extract showed presence of phytol, palmitic acid, linoleic acid, 1,2-benzenedicarboxylic acid, mono(2ethylhexyl) ester, DL- $\alpha$-tocopherol acetate, $\beta$-amyrone, 12-hydroxy-8,10-heptadecadienoic acid and stigmast-4-en-3one (Table 4; Figure 4).

Table 1 Phytochemical compounds in the benzene leaf extract of Abelmoschus manihot

\begin{tabular}{llll}
\hline $\begin{array}{l}\text { Compound } \\
\text { Name }\end{array}$ & $\begin{array}{l}\text { Retention } \\
\text { Time }\end{array}$ & $\begin{array}{l}\text { Molecular } \\
\text { Weight } \mathbf{( g / m o l )}\end{array}$ & $\begin{array}{l}\text { Molecular } \\
\text { Formula }\end{array}$ \\
\hline Phytol & 16.514 & 296.531 & Structure \\
\hline Palmitic acid & 18.024 & 256.424 &
\end{tabular}

\begin{tabular}{llll}
\hline Linoleic acid & 19.620 & 280.445 & $\mathrm{C}_{18} \mathrm{H}_{32} \mathrm{O}_{2}$
\end{tabular}

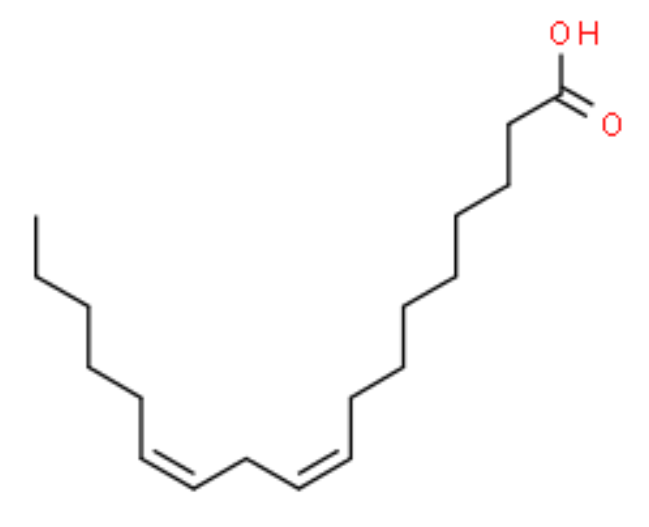




\begin{tabular}{llll}
\hline Dioctyl phthalate & 22.756 & 390.556 & $\mathrm{C}_{24} \mathrm{H}_{38} \mathrm{O}_{4}$
\end{tabular}<smiles>CCCCCCCCCCOC(=O)c1ccccc1C(=O)OCCCCCC</smiles>

\begin{tabular}{llll}
\hline Tocopherol & 27.183 & 430.706 & $\mathrm{C}_{29} \mathrm{H}_{50} \mathrm{O}_{2}$
\end{tabular}
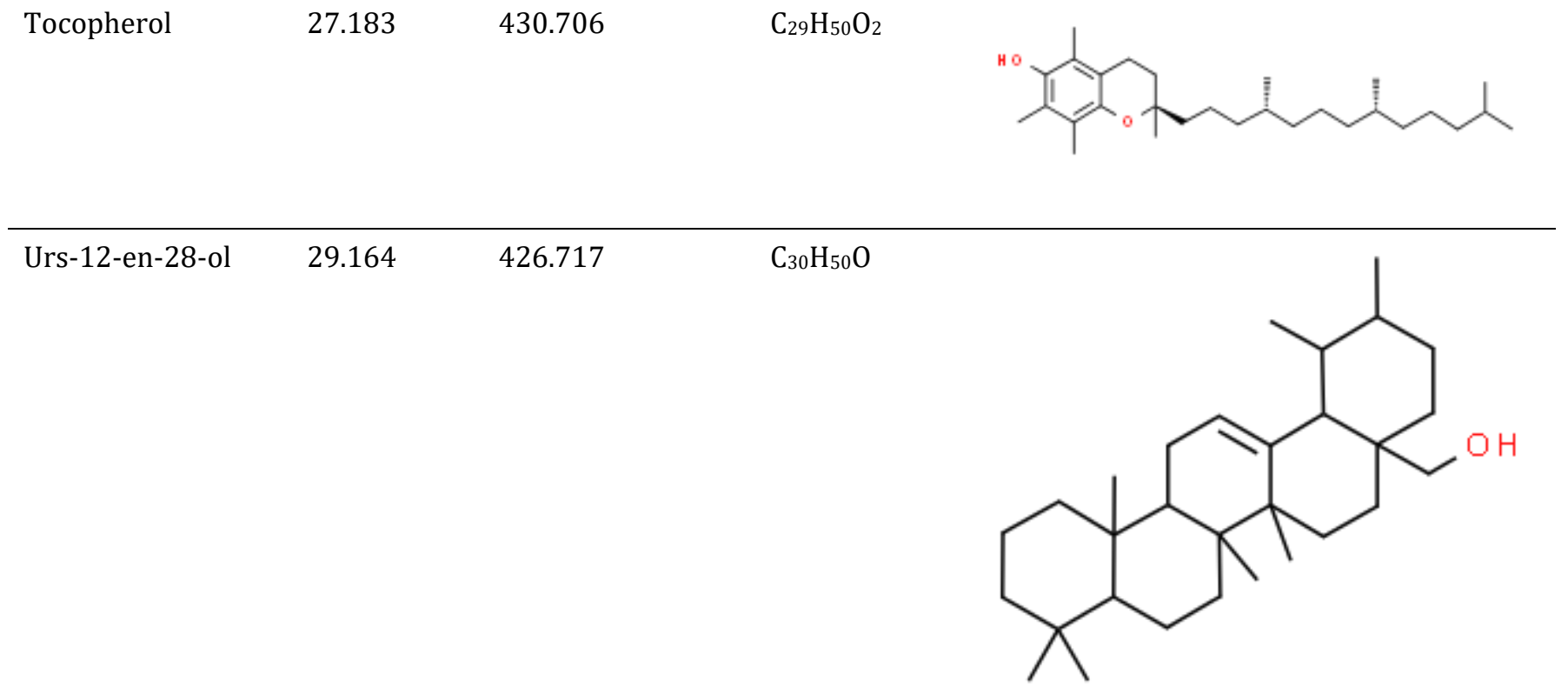

(2E,4E)-2,4-

29.739

266.419

$\mathrm{C}_{17} \mathrm{H}_{30} \mathrm{O}_{2}$

heptadecadienoic

acid

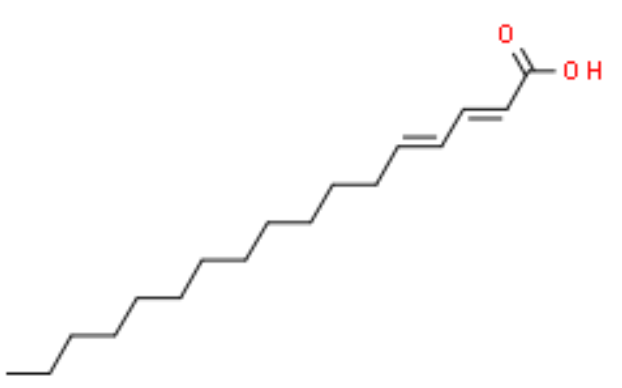

\begin{tabular}{llll}
\hline Stigmast-4-en-3- & 30.839 & 412.690 & $\mathrm{C}_{29} \mathrm{H}_{48} \mathrm{O}$
\end{tabular}

one

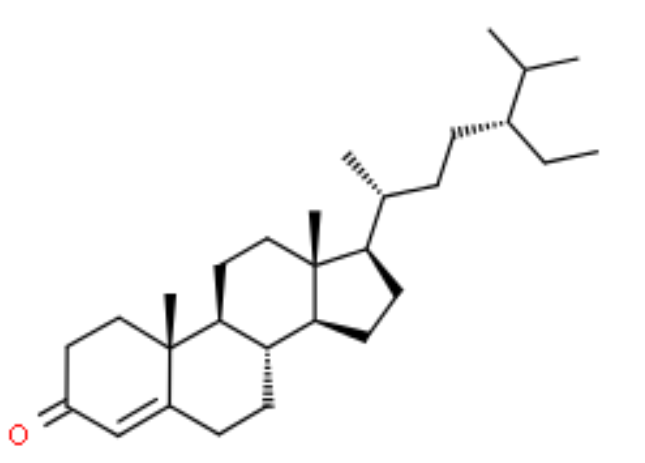




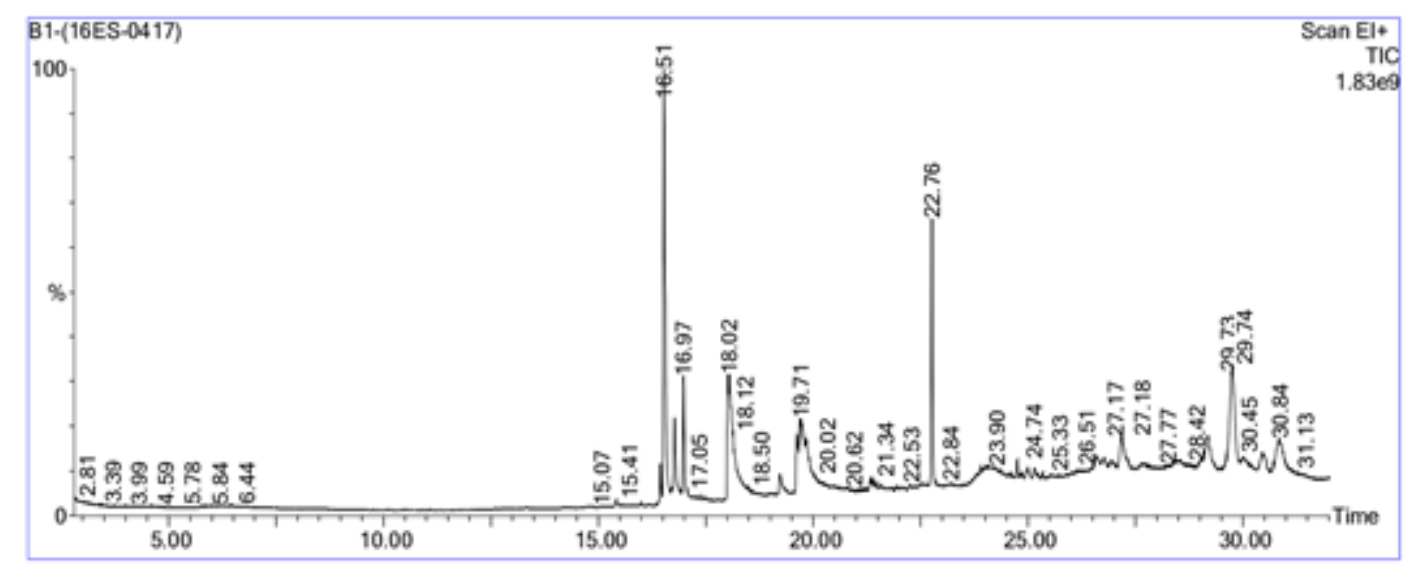

Figure 1 GC-MS chromatogram of benzene leaf extract of Abelmoschus manihot

\begin{tabular}{lllllll}
\hline$\#$ & RT & Scan & Height & Area & Area \% & Norm \% \\
\hline 1 & 16.514 & 2742 & $1,716,226,304$ & $76,951,480.0$ & 18.433 & 100.00 \\
2 & 16.769 & 2793 & $292,744,608$ & $12,344,304.0$ & 2.957 & 16.04 \\
3 & 16.969 & 2833 & $475,076,000$ & $17,730,502.0$ & 4.247 & 23.04 \\
4 & 18.024 & 3044 & $507,284,352$ & $73,028,112.0$ & 17.493 & 94.90 \\
5 & 19.620 & 3363 & $236,198,192$ & $13,875,433.0$ & 3.324 & 18.03 \\
6 & 19.705 & 3380 & $295,806,080$ & $59,129,820.0$ & 14.164 & 76.84 \\
7 & 22.756 & 3990 & $1,090,984,832$ & $37,132,468.0$ & 8.895 & 48.25 \\
8 & 27.183 & 4875 & $124,673,560$ & $16,795,720.0$ & 4.023 & 21.83 \\
9 & 29.164 & 5271 & $134,499,904$ & $23,895,588.0$ & 5.724 & 31.05 \\
10 & 29.739 & 5386 & $411,425,792$ & $57,607,016.0$ & 13.799 & 74.86 \\
11 & 30.839 & 5606 & $138,842,016$ & $28,982,470.0$ & 6.942 & 37.66 \\
\hline
\end{tabular}

Table 2 Phytochemical compounds in the chloroform leaf extract of Abelmoschus manihot

\begin{tabular}{lllll}
\hline $\begin{array}{l}\text { Compound } \\
\text { Name }\end{array}$ & $\begin{array}{l}\text { Retention } \\
\text { Time }\end{array}$ & $\begin{array}{l}\text { Molecular } \\
\text { Weight }(\mathbf{g} / \mathbf{m o l})\end{array}$ & $\begin{array}{l}\text { Molecular } \\
\text { Formula }\end{array}$ & Structure \\
\hline Phytol & 16.514 & 296.531 & $\mathrm{C}_{20} \mathrm{H}_{40} \mathrm{O}$ & \\
\hline Methyl isopalmitate & 17.504 & 270.45 & $\mathrm{C}_{17 \mathrm{H}_{34} \mathrm{O}_{2}}$ &
\end{tabular}




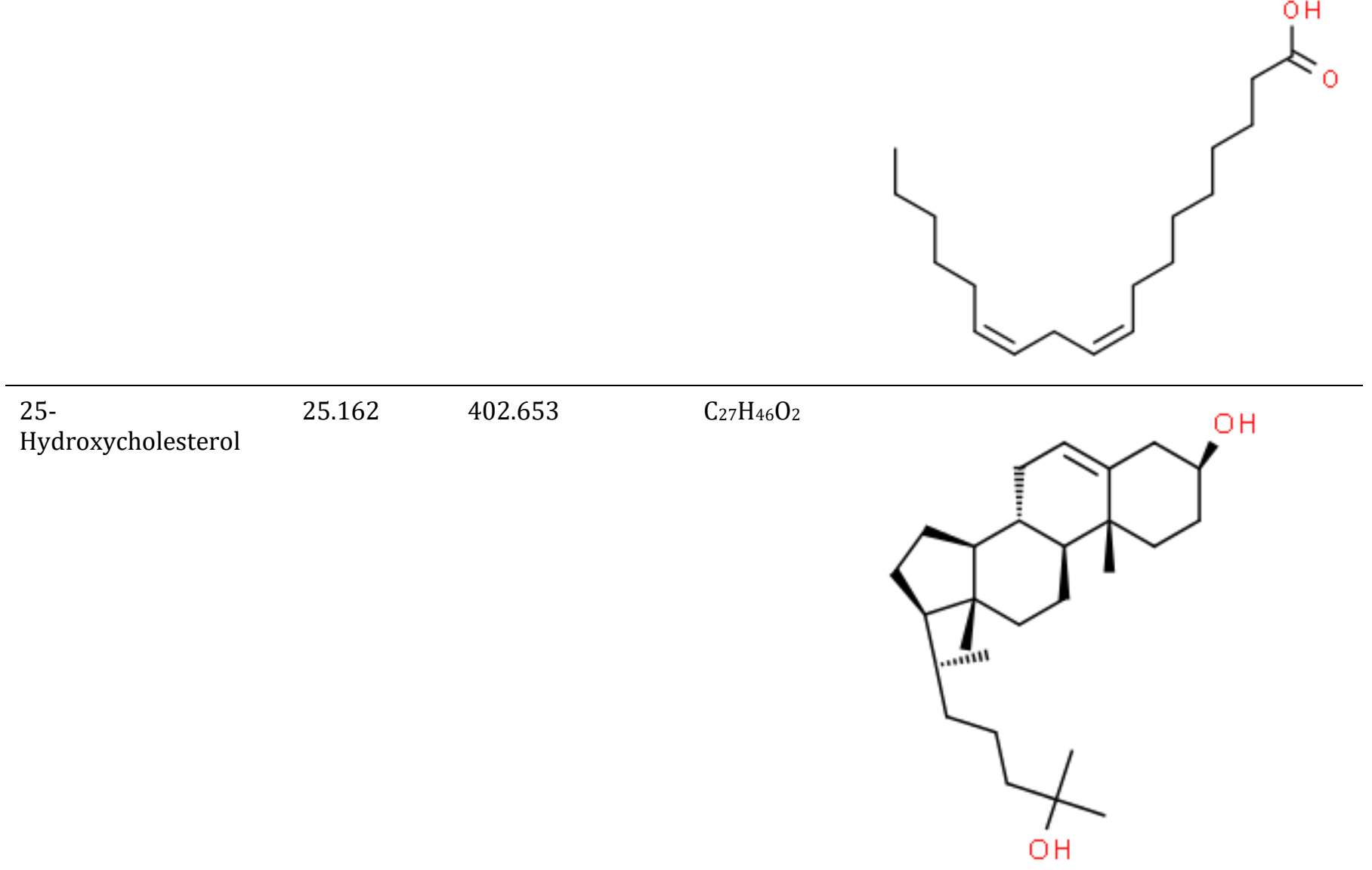

\section{DL- $\alpha$-tocopherol} acetate
27.193

472.743

$\mathrm{C}_{31} \mathrm{H}_{52} \mathrm{O}_{3}$

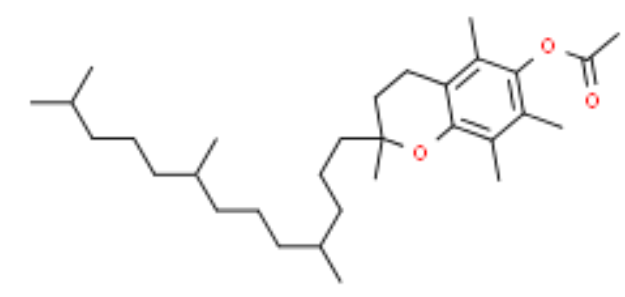

3,5-Di-tert-

butylbenzaldehyde

29.184

218.335

$\mathrm{C}_{15} \mathrm{H}_{22} \mathrm{O}$

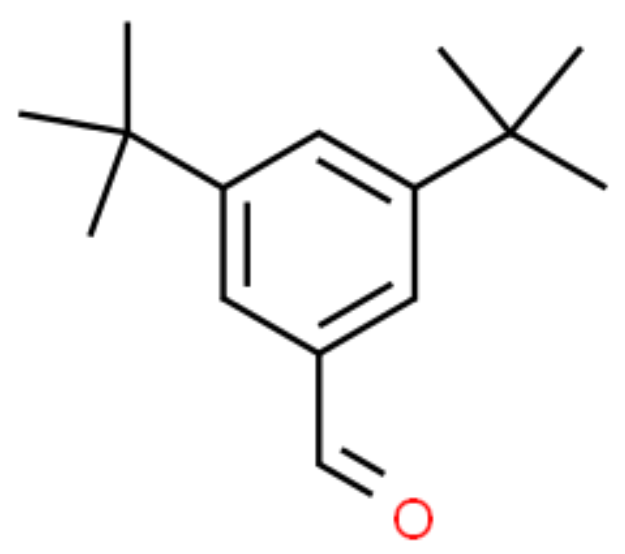

12-hydroxy-8,10-

heptadecadienoic acid
282.418

29.759

282.418

$\mathrm{C}_{17} \mathrm{H}_{30} \mathrm{O}_{3}$<smiles>CCCCCC(O)/C=C/C=C/CCCCCCC(=O)O</smiles> 
22,23-

dibromostigmasterol acetate
$30.494 \quad 614.536$

614.536
$\mathrm{C}_{31} \mathrm{H}_{50} \mathrm{Br}_{2}$

$\mathrm{O}_{2}$<smiles>CCC(C(C)C)C(Br)C(Br)C(C)C1CCC2C3CC=C4CC(OC(C)=O)CCC4(C)C3CCC12C</smiles>

\begin{tabular}{llll}
\hline Cholest-4-en-3-one & 30.909 & 384.638 & $\mathrm{C}_{27} \mathrm{H}_{44} \mathrm{O}$
\end{tabular}<smiles>CC(C)CCC[C@H](C)[C@H]1CC[C@H]2[C@@H]3CCC4=CC(=O)CC[C@]4(C)[C@H]3CC[C@@]21C</smiles>

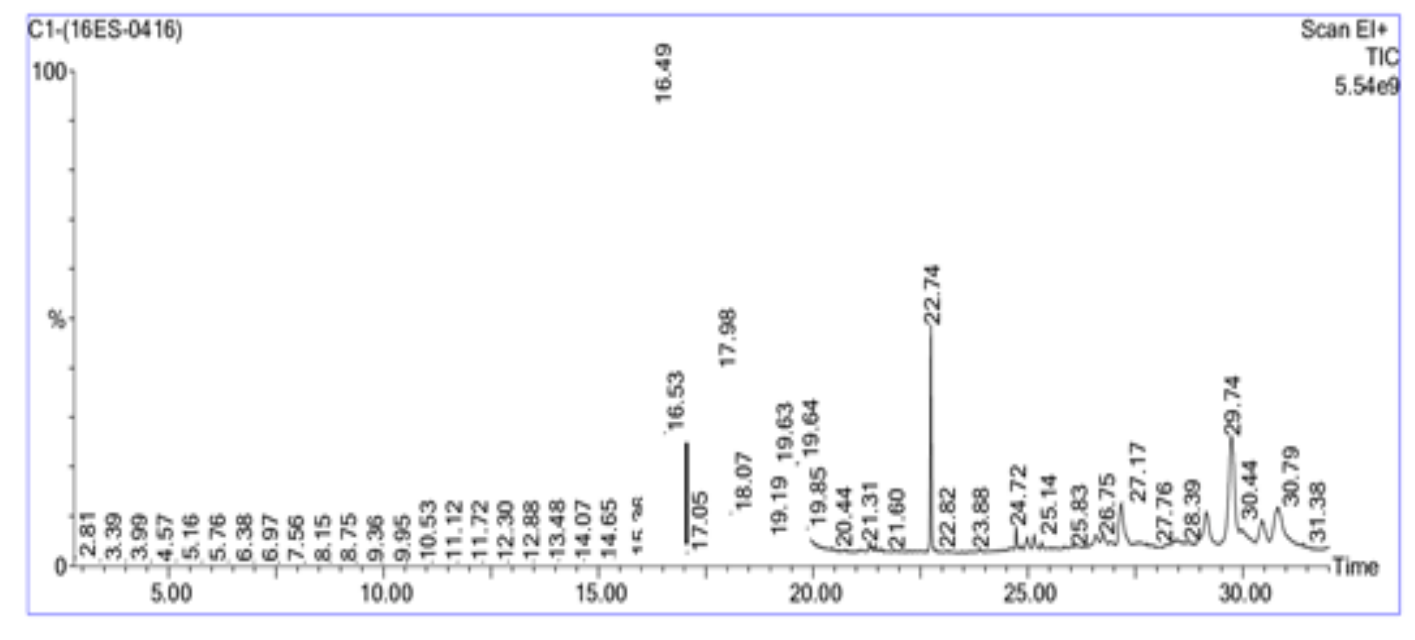

Figure 2 GC-MS chromatogram of chloroform leaf extract of Abelmoschus manihot

\begin{tabular}{lllllll}
\hline$\#$ & RT & Scan & Height & Area & Area \% & Norm \% \\
\hline 1 & 16.494 & 2738 & $5,332,435,968$ & $199,765,216.0$ & 18.764 & 100.00 \\
2 & 16.749 & 2789 & $760,535,616$ & $25,432,190.0$ & 2.389 & 12.73 \\
3 & 16.949 & 2829 & $1,245,597,696$ & $43,947,284.0$ & 4.128 & 22.00 \\
4 & 17.979 & 3035 & $2,091,796,352$ & $151,928,192.0$ & 14.271 & 76.05 \\
5 & 19.555 & 3350 & $676,800,768$ & $38,003,944.0$ & 3.570 & 19.02 \\
6 & 19.635 & 3366 & $989,754,816$ & $160,485,808.0$ & 15.075 & 80.34 \\
7 & 22.736 & 3986 & $2,544,867,840$ & $80,274,888.0$ & 7.540 & 40.18 \\
8 & 27.168 & 4872 & $446,307,072$ & $56,145,404.0$ & 5.274 & 28.11 \\
9 & 29.154 & 5269 & $365,878,112$ & $59,010,672.0$ & 5.543 & 29.54 \\
10 & 29.739 & 5386 & $1,129,086,336$ & $164,345,792.0$ & 15.437 & 82.27 \\
11 & 30.819 & 5602 & $383,930,848$ & $85,270,976.0$ & 8.010 & 42.69 \\
\hline
\end{tabular}


Table 3 Phytochemical compounds in the methanol leaf extract of Abelmoschus manihot

\begin{tabular}{llll}
\hline $\begin{array}{l}\text { Compound } \\
\text { Name }\end{array}$ & $\begin{array}{l}\text { Retention } \\
\text { Time }\end{array}$ & $\begin{array}{l}\text { Molecular } \\
\text { Weight } \\
\text { (g/mol) }\end{array}$ & $\begin{array}{l}\text { Molecular } \\
\text { Formula }\end{array}$ \\
\hline Phytol & 16.514 & 296.531 & Structure \\
\hline
\end{tabular}

2,3-Dimethyl-8-oxo-non- 16.694
2-enal

Palmitic acid 18.024

\begin{tabular}{llll}
\hline 1,1'-bi(cyclohexyl) & 19.635 & 166.303 & $\mathrm{C}_{12} \mathrm{H}_{22}$
\end{tabular}

\begin{tabular}{llll}
\hline $\mathrm{DL}-\alpha$-tocopherol acetate & 27.193 & 472.743 & $\mathrm{C}_{31} \mathrm{H}_{52} \mathrm{O}_{3}$
\end{tabular}

Urs-12-en-3-ol, acetate, $29.194 \quad 468.754 \quad \mathrm{C}_{32} \mathrm{H}_{52} \mathrm{O}_{2}$

(3.ß.)-

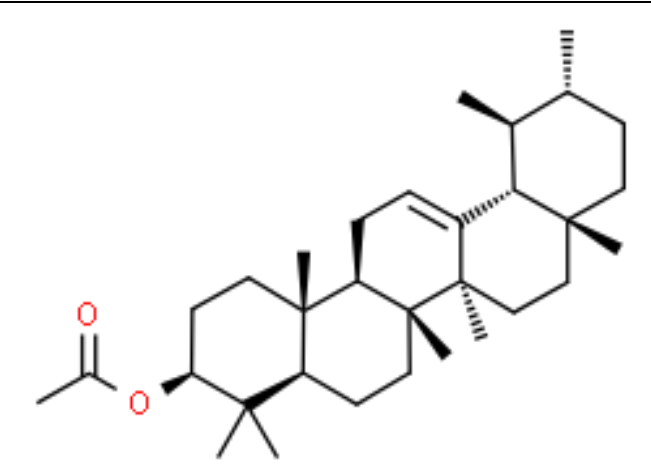




\begin{tabular}{llll}
\hline Fludrocortisone acetate & 30.910 & 422.487 & $\mathrm{C}_{23} \mathrm{H}_{31} \mathrm{FO}_{6}$
\end{tabular}
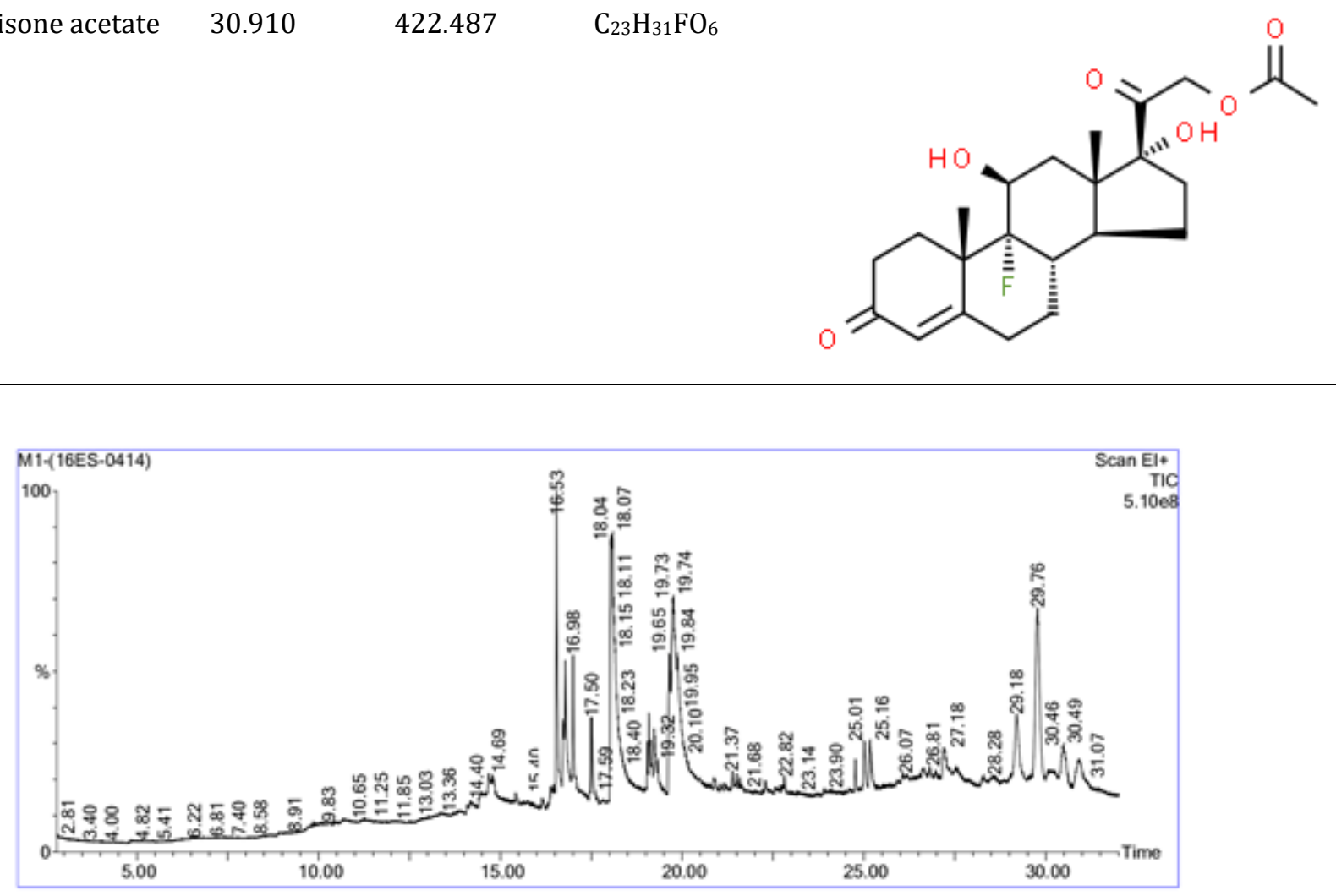

Figure 3 GC-MS chromatogram of methanol leaf extract of Abelmoschus manihot

\begin{tabular}{lllllll}
\hline$\#$ & RT & Scan & Height & Area & Area \% & Norm \% \\
\hline 1 & 16.534 & 2746 & $420,916,928$ & $16,048,945.0$ & 6.381 & 23.95 \\
2 & 16.789 & 2797 & $121,285,480$ & $4,035,635.5$ & 1.605 & 6.02 \\
3 & 16.984 & 2836 & $176,739,520$ & $5,988,394.5$ & 2.381 & 8.94 \\
4 & 17.504 & 2940 & $116,851,536$ & $5,817,687.5$ & 2.313 & 8.68 \\
5 & 18.069 & 3053 & $377,771,200$ & $66,083,348.0$ & 26.275 & 98.61 \\
6 & 19.650 & 3369 & $196,293,456$ & $10,834,127.0$ & 4.308 & 16.17 \\
7 & 19.740 & 3387 & $276,188,416$ & $67,011,896.0$ & 26.644 & 100.00 \\
8 & 25.162 & 4471 & $65,625,952$ & $4,790,231.0$ & 1.905 & 7.15 \\
9 & 27.193 & 4877 & $42,989,608$ & $5,609,520.5$ & 2.230 & 8.37 \\
10 & 29.184 & 5275 & $94,510,936$ & $14,102,153.0$ & 5.607 & 21.04 \\
11 & 29.759 & 5390 & $243,846,832$ & $34,815,536.0$ & 13.843 & 51.95 \\
12 & 30.494 & 5537 & $52,523,988$ & $7,831,631.5$ & 3.114 & 11.69 \\
13 & 30.909 & 5620 & $39,656,244$ & $8,534,596.0$ & 3.393 & 12.74 \\
\hline
\end{tabular}


Table 4 Phytochemical compounds in the ethanol leaf extract of Abelmoschus manihot

\begin{tabular}{llll}
$\begin{array}{l}\text { Compound } \\
\text { Name }\end{array}$ & $\begin{array}{l}\text { Retention } \\
\text { Time }\end{array}$ & $\begin{array}{l}\text { Molecular } \\
\text { Weight } \\
\text { (g/mol) }\end{array}$ & $\begin{array}{l}\text { Molecular } \\
\text { Formula }\end{array}$ \\
\hline Phytol & 296.531 & Structure \\
\hline Palmitic acid & 16.514 & 256.424 & $\mathrm{C}_{16} \mathrm{H}_{32} \mathrm{O}_{2}$
\end{tabular}

Linoleic acid $19.620 \quad 280.445$

1,2-
$\begin{aligned} & \text { benzenedicarboxylic } \\ & \text { acid, } \\ & \text { ethylhexyl) ester }\end{aligned}$

DL- $\alpha$-tocopherol
acetate

B-amyrone 29.154




\begin{tabular}{llll}
\hline $\begin{array}{l}\text { 12-hydroxy-8,10- } \\
\text { heptadecadienoic acid }\end{array}$ & 29.759 & 282.418 & $\mathrm{C}_{17} \mathrm{H}_{30} \mathrm{O}_{3}$
\end{tabular}
heptadecadienoic acid<smiles>CCCCCC(O)C=CC=CCCCCCCC(=O)O</smiles>

\begin{tabular}{llll}
\hline Stigmast-4-en-3-one $\quad 30.839$ & 412.691 & $\mathrm{C}_{29} \mathrm{H}_{48} \mathrm{O}$
\end{tabular}

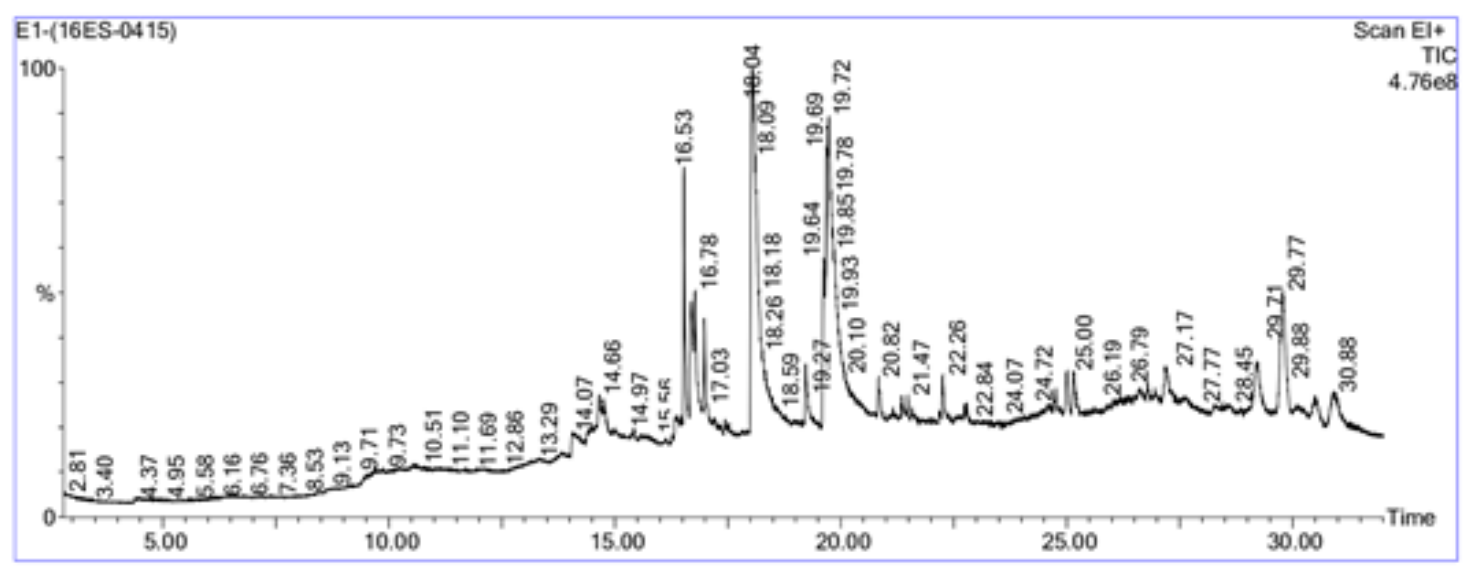

Figure 4 GC-MS chromatogram of ethanol leaf extract of Abelmoschus manihot

\begin{tabular}{lllllll}
\hline$\#$ & RT & Scan & Height & Area & Area \% & Norm \% \\
\hline 1 & 16.529 & 2745 & $274,019,040$ & $10,499,938.0$ & 4.710 & 14.91 \\
2 & 16.694 & 2778 & $129,632,176$ & $10,122,956.0$ & 4.541 & 14.38 \\
3 & 16.779 & 2795 & $139,496,160$ & $9,295,630.0$ & 4.170 & 13.20 \\
4 & 16.969 & 2833 & $109,948,152$ & $4,705,987.0$ & 2.111 & 6.68 \\
5 & 18.040 & 3047 & $385,880,288$ & $65,543,028.0$ & 29.400 & 93.08 \\
6 & 19.215 & 3282 & $64,294,592$ & $4,270,705.5$ & 1.916 & 6.07 \\
7 & 19.635 & 3366 & $174,992,736$ & $8,233,253.0$ & 3.693 & 11.69 \\
8 & 19.715 & 3382 & $322,436,800$ & $70,413,712.0$ & 31.585 & 100.00 \\
9 & 27.193 & 4877 & $37,345,408$ & $4,941,854.0$ & 2.217 & 7.02 \\
10 & 29.194 & 5277 & $53,843,832$ & $8,319,476.0$ & 3.732 & 11.82 \\
11 & 29.769 & 5392 & $128,888,256$ & $18,541,098.0$ & 8.317 & 26.33 \\
12 & 30.910 & 5620 & $37,380,732$ & $8,044,664.5$ & 3.609 & 11.42 \\
\hline
\end{tabular}




\section{Discussion}

Plants are a rich source of bioactive phytochemicals which provide health benefits for humans further than those attributed to macronutrients and micronutrients. Todarwal et al. [20] have reviewed the ethnobotany, phytochemistry and pharmacological properties of Abelmoschus manihot. This plant is known for secondary metabolites like flavonoids [21] and steroids [12]. The phytochemical constituents obtained via GC-MS from different solvent leaf extracts of Abelmoschus manihot from the present study were found to have various biological properties reported elsewhere, viz., antimicrobial, anti-inflammatory, antioxidant, antidiuretic, antifungal, antieczemic, antiacne, antiarthritic, anticoronary, antiseptic, antidermatic, antispasmodic, antibronchitic, antidiabetic, antiandrogenic, antitumour, hypocholesterolemic, hepatoprotective, hypoglycemic, lubricant, nematicide and pesticide. Besides these, this plant is popular for its young, tender, juicy pods which can be consumed in different forms like boiled, fried or cooked $[22,23]$. High protein source due to high lysine level in seeds make this plant as an alternative to soybean and therefore could be used as a supplement to cereal based diets [24, 25]. In medical application, it has been found as a good component for plasma replacement or blood volume expander [26-29]. It also has been reported as medicine for the control of fertility, childbirth and also to act as a stimulator in milk production for lactating mothers [30-33].

\section{Conclusion}

Studies on phytoprinciples from Abelmoschus manihot need to be evaluated in a scientific manner so as to identify potential lead compounds for further development, as ethnobotanical and traditional uses of natural compounds, especially those of plant origin, are often very effective and generally believed to be safe for human use.

\section{Compliance with ethical standards}

\section{Acknowledgments}

The authors thank the Chemistry division, School of Advanced Science, VIT University, Vellore, Tamil Nadu, India in providing their help with regard to GC-MS analysis.

\section{Disclosure of conflict of interest}

The authors declare that there is no conflict of interest.

\section{References}

[1] Charrier A. (1984). Genetic resources of the genus Abelmoschus Med. (Okra). International Board for Plant Genetic Resources, Rome, Italy.

[2] Grubben GJH. (1977). Tropical vegetables and their genetic resources. (H.D. Tindall and J.T. Williams, eds.) IBPGR, Rome, 197.

[3] Onakpa MM. (2013). Ethnomedicinal, phytochemical and pharmacological profile of genus Abelmoschus. Phytopharmacology, 4(3), 648-663.

[4] Patil P, Sutar S, John JK, Malik S, Rao S, Yadav S and Bhat KV. (2015). A systematic review of the genus Abelmoschus (Malvaceae). Rheedea, 25(1), 14-30.

[5] Chevalier A. (1940). L'origine, la culture et les usages de cinq Hibiscus de la section Abelmoschus. Review of Botanical Applications, 20, 319-328.

[6] Hauman L. (1963). La Flore du Congo, du Rwanda et du Burundi, 142-145.

[7] Wang Y, Wang B, Lu B, Li P, Yang B, Ji W and Meng Q. (2015). Analysis of volatile constituents from Abelmoschus manihot by GC-MS. Asian Journal of Chemistry, 27(10), 3684-3686.

[8] Han XL and Situ ML. (1997). 82 cases of canker treated with cataplasm made from the flower of Abelmoschus manihot. Clinical Journal of Chinese Traditional Medicine, 9, 308.

[9] Zhang SH. (1997). Outer treatment of small area burn applied with oil of Abelmoschus manihot. Lishizhen Medicine and Material Medica Research, 8, 96-103. 
[10] Chen D. (2001). Effect observation of Huangkuai capsule on chronic nephritis. Chinese Journal of Integrative and Traditional Western Nephrology, 2, 299-300.

[11] Jain PS, Todarwal AA, Bari SB and Surana JS. (2011). Analgesic activity of Abelmoschus manihot extracts. International Journal of Pharmacology, 7, 505-520.

[12] Jain PS and Bari SB. (2009). Isolation of Stigmasterol and $\gamma$-Sitosterol from petroleum ether extract of woody stem of Abelmoschus manihot. Asian Journal of Biological Sciences, 2, 112-117.

[13] Lin-lin WU, Yang X, Huang Z, Liu H and Guang WU. (2007). In vivo and in vitro antiviral activity of hyperoside extracted from Abelmoschus manihot L. Medic. Acta Pharmacologica Sinica, 28, 404-409.

[14] Yao YM. (1994). Inhibition effect of the flower of Abelmoschus manihot extraction on Diplococcus gonorrhoeae. Jiangsu Journal of Traditional Chinese Medicine, 15, 43-49.

[15] Gu HZ and Song JB. (1998). Progress of studies on Abelmoschus manihot (L.) Medic. Journal of Chinese Medicine Materials, 21, 158-160.

[16] Dua VK, Pandey AC, Alam ME and Dash AP. (2006). Larvicidal activity of Hibiscus abelmoschus Linn. (Malvaceae) against mosquitoes. Journal of the American Mosquito Control Association, 22, 155-157.

[17] Puel C, Mathey J, Davicco MJ, Lebecque P, Chanteranne B, Horcajada MN and Coxam V. (2005). Preventive effect of Abelmoschus manihot (L) Medik on bone loss in the overiectomised rats. Journal of Ethnopharmacology, 99, 655-660.

[18] Cheng XP, Qin S, Dong LY and Zhou JN. (2006). Inhibitory effect of total flavone of Abelmoschus manihot L. Medic on NMDA receptor-mediated current in cultured rat hippocampal neurons. Neuroscience Resources, 55, 142145 .

[19] Vogel. (1978). Textbook of practical organic chemistry, London, 1368.

[20] Todarwal A, Jain P and Bari S. (2011). Abelmoschus manihot Linn: ethnobotany, phytochemistry and pharmacology. Asian Journal of Traditional Medicines, 6(1), 1-7.

[21] Xian YL, Zhao YY and Liang H. (2007). A flavonoid glucuronide from Abelmoschus manihot (L.) Medik. Biochemical Systematics and Ecology, 35(12), 891-893.

[22] Ndunguru J and Rajabu AC. (2004). Effect of okra mosaic virus disease on the above-ground morphological yield components of okra in Tanzania. Scientia Horticulturae, 99, 225-235.

[23] Akintoye HA, Adebayo AG and Aina 00. (2011). Growth and yield response of okra intercropped with live mulches. Asian Journal of Agricultural Research, 5, 146-153.

[24] Karakoltsidis PA and Constantinides SM. (1975). Okra seeds: A new protein source. Journal of Agricultural and Food Chemistry, 23(6), 1204-1207.

[25] Al-Wandawi H. (1983). Chemical composition of seeds of two okra cultivars. Journal of Agricultural and Food Chemistry, 31(6), 1355-1358.

[26] Savello PH, Martin FW and Hill JM. (1980). Nutritional composition of okra seed meal. Journal of Agricultural and Food Chemistry, 28, 1163-1166.

[27] Markose BL and Peter KV. (1990). Okra: Review of research on vegetable and tuber crops. Kerala Agricultural University Press, Kerala, India.

[28] Lengsfeld C, Titgemeyer F, Faller G and Hensel A. (2004). Glycosylated compounds from okra inhibit adhesion of Helicobacter pylori to human gastric mucosa. Journal of Agricultural and Food Chemistry, 52, 1495-1503.

[29] Kumar S, Dagnoko S, Haougui A, Ratnadass A, Pasternak D and Kouame C. (2010). Okra (Abelmoschus spp.) in West and Central Africa: potential and progress on its improvement. African Journal of Agricultural Research, 5, 3590-3598.

[30] Powell JM. (1976). Ethnobotany. Part III of New Guinea Vegetation (K. P. Paijmans, ed.) CISRO/ ANU Press, Canberra, Australia, 106-183.

[31] Perry L. (1980). Medicinal plants of east and southeast Asia- attributed properties and uses. MIT Press, Cambridge, Massachusetts.

[32] Bourdy G and Walter A. (1992). Maternity and medicinal plants in Vanuatu. I. The cycle of reproduction. Journal of Ethnopharmacology, 37, 179-196. 
[33] Salomon-Nekiriai C. (1995). Medical knowledge and know-how to kanak women. ESK/CORDET, Noumea, New Caledonia.

\section{How to cite this article}

Divya S, Arivoli S and Tennyson S. (2020). GC-MS analysis of Abelmoschus manihot (L.) Medik (Malvaceae) leaves. World Journal of Advanced Research and Reviews, 5(2), 67-79. 